MyoD1, CD117, PAX8, TTF1, inhibin, HepPar1, arginase, Glypican-3, pan-TRK, NUT, ALK1, ROS1, synaptophysin, chromogranin, and INSM1. Ki-67 labelling index was estimated at up to $30 \%$. Excision of the tumour appeared focally incomplete. Two lymph nodes in the right neck dissection were involved by direct extension.

Electron microscopy on glutaraldehyde-fixed tumour tissue confirmed fibroblastic differentiation. The tumour cells showed abundant cytoplasm which contained prominent, dilated rough endoplasmic reticulum (Fig. 2E). Some cells showed prominent Golgi apparatus (Fig. 2F). The cells had frequent slender cytoplasmic projections intimately associated with collagen fibrils (Fig. 2F). Collections of poorly preserved mitochondria were present in occasional cells. Scattered lysosomes, lipid droplets and glycogen particles were present. Isolated cells contained vague intermediate filaments. No peripheral densities were seen. The nuclei showed prominent nucleoli.

Cytogenetic analysis revealed a complex karyotype including rearrangements of $1 \mathrm{p}$ and $10 \mathrm{q}$ raising the possibility of a $\mathrm{t}(1 ; 10)(\mathrm{p} 22 ; \mathrm{q} 24)$ translocation.

Our case report highlights the diagnostic challenges and potential pitfall for misdiagnosis in a poorly differentiated head and neck high grade epithelioid/sarcomatoid malignancy with PRAME expression. While conventional (low grade) areas were evident on the resection, the initial core biopsies were exclusively composed of the peripheral high grade areas which raised the possibility of malignant melanoma. Interestingly, PRAME expression was observed only in the high grade (poorly differentiated/undifferentiated) areas. This finding, which has not been explicitly noted in previous studies of PRAME-positive high grade MIFS, is in keeping with our current understanding of acral (low grade) MIFS which are negative for PRAME expression. We performed PRAME immunohistochemistry on two historical cases of acral MIFS which were negative (data not shown). PRAME is an immunogenic tumour-associated antigen which is expressed in diverse non-melanocytic epithelial, haematological and soft tissue malignancies. ${ }^{11}$ Its potential as an immunotherapeutic target of prognostic utility awaits further research. ${ }^{12}$

In summary, we present a case of a rare head and neck high grade MIFS in an elderly male patient, which subsequently progressed to multi-site metastatic disease despite adjuvant therapy. High grade MIFS can show PRAME immunoexpression and mimic malignant melanoma. Tissue diagnosis rests on the histological identification of the conventional (low grade) areas which are characteristic, but can be under-sampled in core biopsies.

Acknowledgement: We thank Dr Tristan Rutland for assistance with scanning of digital slides.

Conflicts of interest and sources of funding: The authors state that there are no conflicts of interest to disclose.

Jeremy N. Pulvers ${ }^{1}$, Samuel T. Roberts ${ }^{2,3}$, Andrew Wignall ${ }^{2}$, Renee C. F. Chan ${ }^{4}$, Anita Muljono ${ }^{5}$, Christopher W. Toon ${ }^{1}$

${ }^{1}$ NSW Health Pathology, Department of Anatomical Pathology, Royal North Shore Hospital, Sydney, NSW, Australia; ${ }^{2}$ Department of Otolaryngology, Head and Neck
Surgery, Royal North Shore Hospital, Sydney, NSW, Australia; ${ }^{3}$ University of Newcastle, Callaghan, NSW, Australia; ${ }^{4}$ Department of Anatomical Pathology, Electron Microscopy Unit, Concord Repatriation General Hospital, Sydney, NSW, Australia; ${ }^{5}$ Histopathology Department, Douglass Hanly Moir Pathology, Macquarie Park, NSW, Australia

Contact: Dr Christopher Toon.

E-mail: christopher.toon@health.nsw.gov.au

1. Montgomery EA, Devaney KO, Giordano TJ, et al. Inflammatory myxohyaline tumor of distal extremities with virocyte or ReedSternberg-like cells: a distinctive lesion with features simulating inflammatory conditions, Hodgkin's disease, and various sarcomas. Mod Pathol 1998; 11: 384-91.

2. Meis-Kindblom JM, Kindblom LG. Acral myxoinflammatory fibroblastic sarcoma: a low-grade tumor of the hands and feet. Am J Surg Pathol 1998; 22: 911-24.

3. Michal M. Inflammatory myxoid tumor of the soft parts with bizarre giant cells. Pathol Res Pract 1998; 194: 529-33.

4. Laskin WB, Fetsch JF, Miettinen M. Myxoinflammatory fibroblastic sarcoma: a clinicopathologic analysis of 104 cases, with emphasis on predictors of outcome. Am J Surg Pathol 2014; 38: 1-12.

5. Hallor KH, Sciot R, Staaf J, et al. Two genetic pathways, $\mathrm{t}(1 ; 10)$ and amplification of $3 \mathrm{p} 11-12$, in myxoinflammatory fibroblastic sarcoma, haemosiderotic fibrolipomatous tumour, and morphologically similar lesions. J Pathol 2009; 217: 716-27.

6. Antonescu CR, Zhang L, Nielsen GP, et al. Consistent $\mathrm{t}(1 ; 10)$ with rearrangements of TGFBR3 and MGEA5 in both myxoinflammatory fibroblastic sarcoma and hemosiderotic fibrolipomatous tumor. Genes Chromosomes Cancer 2011; 50: 757-64.

7. Suster D, Michal M, Huang H, et al. Myxoinflammatory fibroblastic sarcoma: an immunohistochemical and molecular genetic study of 73 cases. Mod Pathol 2020; 33: 2520-33.

8. Michal M, Kazakov DV, Hadravsky L, et al. High-grade myxoinflammatory fibroblastic sarcoma: a report of 23 cases. Ann Diagn Pathol 2015; 19: 157-63.

9. Vroobel K, Miah A, Fisher C, et al. Myxoinflammatory fibroblastic sarcoma of the scalp: aggressive behavior at a rare, nonextremity site. Int J Surg Pathol 2015; 23: 292-7.

10. McFarlane R, Meyers AD, Golitz L. Myxoinflammatory fibroblastic sarcoma of the neck. J Cutan Pathol 2005; 32: 375-8.

11. Lezcano C, Jungbluth AA, Nehal KS, et al. PRAME expression in melanocytic tumors. Am J Surg Pathol 2018; 42: 1456.

12. Roszik J, Wang WL, Livingston JA, et al. Overexpressed PRAME is a potential immunotherapy target in sarcoma subtypes. Clin Sarcoma Res 2017; 7: 1-7.

DOI: https://doi.org/10.1016/j.pathol.2021.07.014

\section{Well-differentiated neuroendocrine tumour arising from diffuse ganglioneuromatosis: first reported case}

To the Editor,

This is the first reported case of a well-differentiated neuroendocrine tumour (WDNET) arising from diffuse ganglioneuromatosis in a patient with neurofibromatosis type 1 (NF1 ). The case additionally highlights diffuse neuroendocrine cell micronests, which may represent a potential precursor lesion from which the WDNET arose.

A 51-year-old female with NF-1 underwent a screening colonoscopy, which revealed a $1.8 \mathrm{~cm}$ mass in the upper rectum. Endoscopy revealed a mass on the posterior wall of the upper rectum, suspicious for a neurofibroma. Biopsy revealed a 
WDNET arising in a ganglioneuroma. A segmental resection was performed, and sections revealed a $0.1 \mathrm{~cm}$ focus of residual low-grade WDNET intimately admixed within a larger area of diffuse ganglioneuromatosis (DGN) (Fig. 1). The WDNET superficially invaded the submucosa and was staged as pT1N0 with 18 negative lymph nodes. Numerous microscopic clusters of neuroendocrine cells were also scattered throughout the lamina propria of the larger DGN (Fig. 2). Twenty months after initial diagnosis and 7 months after resection, a follow up colonoscopy confirmed no residual disease.

NF-1 involves the gastrointestinal tract in 5-25\% of affected individuals. ${ }^{1}$ Gastrointestinal stromal tumours are the most common manifestation, but WDNETs of the duodenum and periampullary region and ganglioneuromas have also been described. Ganglioneuromas may be subclassified into (1) polypoid ganglioneuroma, (2) ganglioneuromatous polyposis, and (3) DGN. ${ }^{2}$ DGN are usually incidental findings in patients with PTEN hamartoma tumour syndrome, multiple endocrine neoplasia syndrome type $2 \mathrm{~B}$, and (less commonly) NF-1. Surgical resection is performed for patients with changes in bowel habit or occlusive episodes due to stricture formation. Histologically, DGN is a poorly-circumscribed, frequently transmural hamartomatous gastrointestinal lesion composed of spindled and ganglion cells. Though transformation of neurofibromas to malignant peripheral nerve sheath tumours is well documented in NF-1, malignancy associated with ganglioneuromas is extremely rare. A single previous report describes rectal WDNETs in association with ganglioneuromas, though the description of multiple separate WDNETs and multiple discrete ganglioneuromas appears more consistent with ganglioneuromatous polyposis than DGN, and the patient had no history of NF-1. ${ }^{4}$ Therefore, to our knowledge, the current case is the first report of a WDNET arising in association with DGN.

In addition, this is the first case to describe microscopic clusters of neuroendocrine cells in the lamina propria in DGN. Similar proliferations have been described in the setting of inflammatory bowel disease, where they are termed 'neuroendocrine cell micronests', and are not thought to have malignant potential. ${ }^{5}$ However, given the presence of numerous neuroendocrine cell clusters in this case, we hypothesise that in the setting of NF-1 and/or in DGN, neuroendocrine cell clusters in the lamina propria may serve as a precursor of WDNET. While the WDNET in the current case was low grade and low stage, it demonstrated aggressive behaviour, as evidenced by submucosal invasion. Though NF-1 and DGN are rare entities, these novel findings of both WDNET and neuroendocrine cell clusters in the lamina propria should prompt a careful examination for WDNET and microscopic neuroendocrine cell clusters in future cases of DGN.

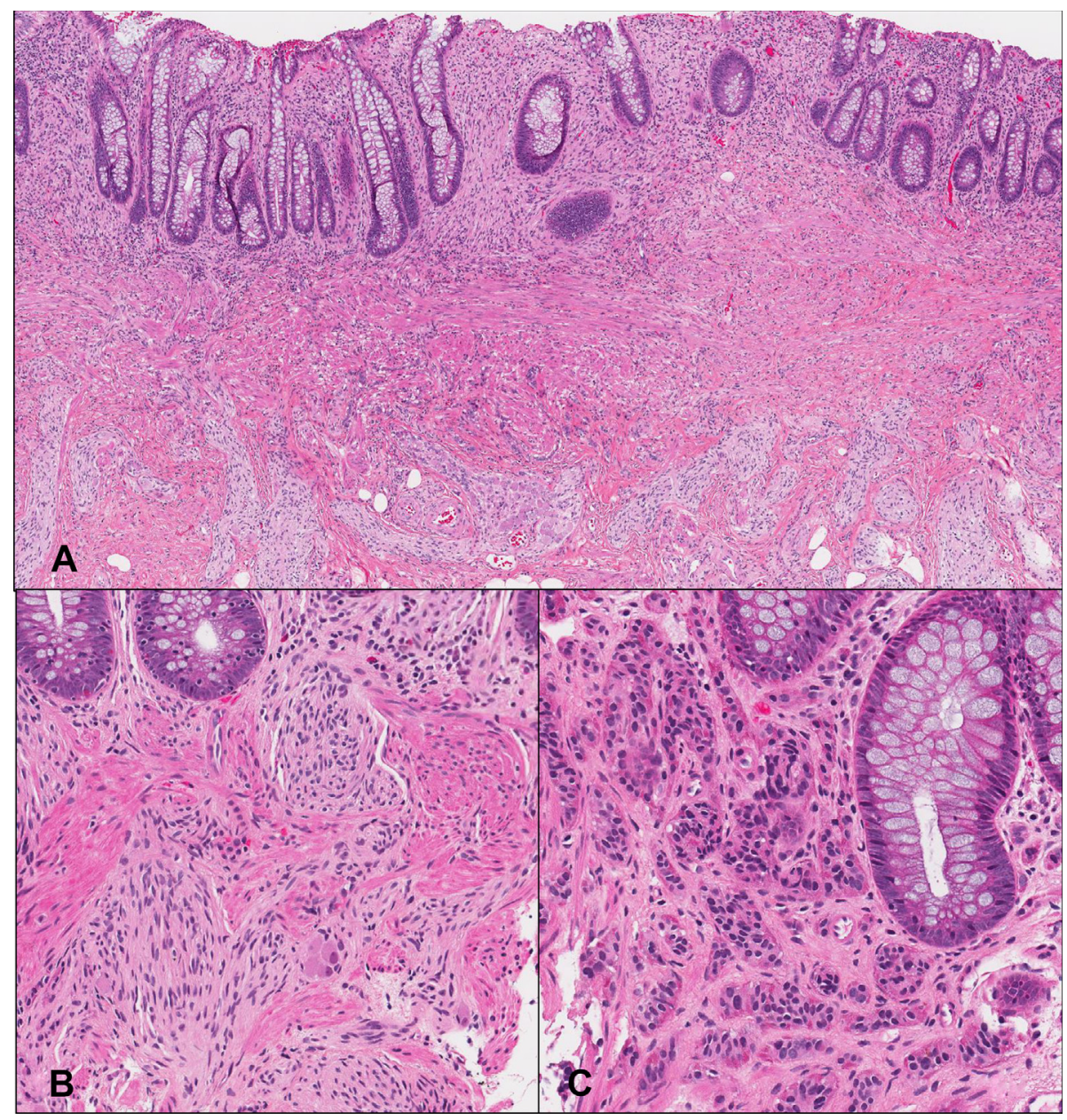

Fig. 1 Residual well-differentiated neuroendocrine tumour (WDNET) associated with diffuse ganglioneuromatosis (DGN). (A) DGN demonstrating a spindle cell proliferation in the lamina propria, disorganised neural and adipose tissue in the submucosa, and interspersed ganglion cells. (B) High-power view of disorganised neural tissue with interspersed ganglion cells. (C) Infiltrative nests of WDNET admixed with the neural tissue of DGN. 


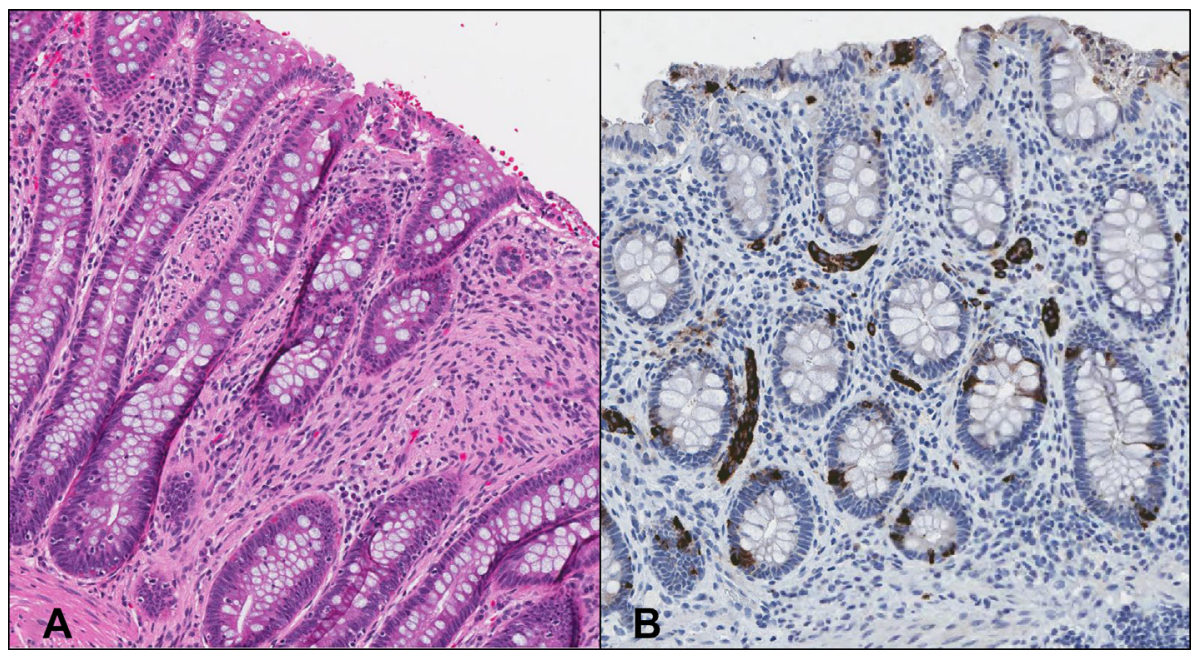

Fig. 2 Diffuse proliferation of neuroendocrine micronests in colonic mucosa. (A) Scattered small nests of neuroendocrine cells in the lamina propria, embedded in the neural tissue of diffuse ganglioneuromatosis, but distant from the neuroendocrine tumour component. (B) Chromogranin immunostain confirms the neuroendocrine nature of the abnormal cell nests in the lamina propria.

Conflicts of interest and sources of funding: The authors state that there are no conflicts of interest to disclose.

\section{Brian K. Cox ${ }^{1}$, Bonnie Balzer ${ }^{1}$, Maha Guindi ${ }^{1}$, Alexandra Gangi ${ }^{2}$, Brent K. Larson ${ }^{1}$}

${ }^{1}$ Department of Pathology and Laboratory Medicine, Cedars-Sinai Medical Center, Los Angeles, CA, USA; ${ }^{2}$ Department of Surgery, Cedars-Sinai Medical Center, Los Angeles, CA, USA

Contact Brian K. Cox, MD.

E-mail: brian.cox2@cshs.org

1. Agaimy A, Vassos N, Croner RS. Gastrointestinal manifestations of neurofibromatosis type 1 (Recklinghausen's disease): clinicopathological spectrum with pathological considerations. Int J Clin Exp Pathol 2012; 5 : $852-62$.

2. Shekitka KM, Sobin LH. Ganglioneuromas of the gastrointestinal tract Relation to von Recklinghausen disease and other multiple tumour syndromes. Am J Surg Pathol 1994; 18: 250-7.

3. Tyway K, Fischer C. Diffuse ganglioneuromatosis in small intestine associated with neurofibromatosis type 1. Ann Diagn Pathol 2009; 13: $50-4$.

4. Haraguchi M, Kinoshita H, Koori M, et al. Multiple rectal carcinoids with diffuse ganglioneuromatosis. World J Surg Oncol 2007; 4: 19.

5. Wong M, Larson BK, Dhall D. Neuroendocrine proliferations in inflammatory bowel disease: differentiating neuroendocrine tumours from neuroendocrine cell micronests. Histopathology 2019; 74: 415-23.

DOI: https://doi.org/10.1016/j.pathol.2021.08.005

\section{High-grade B-cell lymphoma with concurrent expression of CD5 and cyclin D1 and MYC rearrangement}

To the Editor,

High-grade B-cell lymphoma not otherwise specified (HGBL-NOS), according to the World Health Organization (WHO) 2017 Classification, represents a heterogeneous group of extremely aggressive neoplasms defined by blastoid cytology, lacking the double or triple hit (DH/TH) rearrangements of $M Y C$ and $B C L 2$ and/or BCL6 genes. ${ }^{1}$ Rigorous immunohistochemical and molecular characterisation of malignant lymphomas with high-grade blastoid features is critical to distinguish HGBCL-NOS from DH/TH lymphomas, Burkitt lymphoma (BL), lymphoblastic lymphoma (LL) and blastoid variant of mantle cell lymphoma (BVMCL). We describe here a rare case of HGBCL-NOS with $M Y C$ rearrangement only and exceptional coexpression of CD5 and cyclin D1. We discuss the dilemma of differential diagnosis, explaining that this uncommon phenotype represents a new observation, not having been described yet in HGBCL-NOS in the current literature.

A 69-year-old female patient was admitted to St Eugenio Hospital of Rome with severely compromised general conditions, weight loss, fever, and abdominal discomfort. A total body computed tomography (CT) scan detected several bulky nodal masses in the retroperitoneum (Fig. 1A). Neither blood count nor blood smear showed any leukaemic picture or circulating abnormal cells. Therefore, a core needle biopsy was performed. Unfortunately, we could not assess the tissue by flow cytometry. Histopathological examination showed a diffuse infiltration of medium sized tumour cells with scant amphophilic/basophilic cytoplasm, round to oval nuclei with fine chromatin, small single (sometimes centrally located) or multiple basophilic nucleoli. Apoptotic bodies were present without a clear-cut 'starry sky' appearance. Numerous mitotic figures were also detected (Fig. 1B,C), and the proliferation index assessed by $\mathrm{Ki}-67$ immunostaining reached $100 \%$ (Fig. 2). These findings were considered compatible with the so-called blastoid morphology. Immunohistochemical staining revealed the diffuse expression of PAX5, CD20, CD10 and IRF4/MUM1 (Fig. 1); a subset of tumour cells expressed BCL6 (Fig. 1), however staining for TdT and CD34 was negative. The lymphoma cells had diffuse and strong expression of BCL2 (Fig. 1) and exhibited high nuclear immunostaining for c-MYC in approximately $90 \%$ of the cells (Fig. 2). EBV-encoded RNA (EBER) was also negative. Follicular dendritic cell meshwork evaluated by CD23 staining was absent. Surprisingly, both CD5 and cyclin D1 were widely expressed by the neoplastic cells. Of note, 